Relations industrielles

Industrial Relations

\title{
La conciliation des différends du travail
}

\section{I - Cas types}

\section{Jean-Paul Ferland}

Volume 6, numéro 3, juin 1951

URI : https://id.erudit.org/iderudit/1023212ar

DOI : https://doi.org/10.7202/1023212ar

Aller au sommaire du numéro

Éditeur(s)

Département des relations industrielles de l’Université Laval

ISSN

0034-379X (imprimé)

1703-8138 (numérique)

Découvrir la revue

Citer cet article

Ferland, J.-P. (1951). La conciliation des différends du travail : I - Cas types. Relations industrielles / Industrial Relations, 6(3), 79-81.

https://doi.org/10.7202/1023212ar
Résumé de l'article

Nous avons cru intéresser nos lecteurs en demandant à deux conciliateurs diplômés, le premier de Laval et le second de Montréal et de Queen's, qui sont fonctionnaires au ministère provincial du Travail, de donner leurs vues sur le climat de la conciliation dans les districts de Montréal et Québec.
Tous droits réservés @ C Département des relations industrielles de l’Université Laval, 1951
Ce document est protégé par la loi sur le droit d'auteur. L’utilisation des services d'Érudit (y compris la reproduction) est assujettie à sa politique d'utilisation que vous pouvez consulter en ligne.

https://apropos.erudit.org/fr/usagers/politique-dutilisation/ 


\section{La conciliation des différends du travail}

\section{TÉmoignages DE CONCILIATEuRS}

Nous avons cru intéresser nos lecteurs en demandant à deux conciliateurs diplômés, le premier de Laval et le second de Montréal et de Queen's, qui sont fonctionnaires au ministère provincial du Travail, de donner leurs vues sur le climat de la conciliation dans les districts de Montréal et Québec.

\section{I - CAS TYPES}

par Jean-Paul Fertand

La conciliation est une procédure qui se situe entre les négociations directes et l'arbitrage. Elle peut avoir lieu lors des négociations, en vue d'une première convention collective ou lors du renouvellement d'une telle convention, ou encore, lors de la discussion d'un grief survenu au cours de la durée d'une convention.

Nous nous limiterons à l'étude des problèmes pouvant survenir aux deux premières phases.

Cette procédure se fait sous la présidence d'un officier du ministère du Travail appelé (conciliateur . Son rôle consiste à rencontrer les parties et à tenter de les amener à une entente.

Les problèmes qu'il rencontre peuvent porter d'une fois à l'autre sur les mêmes sujets, ce qui ne signifie pas que les moyens de leur apporter une solution sont identiques. En effet, les agents et les circonstances changent toujours. Il faut donc au conciliateur une grande facilité d'adaptation.

Quoique la Loi des relations ouvrières existe depuis le mois de février 1944, il arrive encore parfois qu'un employeur refuse de négocier. La raison qu'il donne est qu'il ne reconnaît pas l'association ouvrière, cornme le représentant autorisé de ses employés, et ceci, bien qu'un cer- tificat ait été émis par la Commission de relations ouvrières. A titre d'exemple, nous citerons le cas suivant: Une association ouvrière est certifiée pour représenter tous les employés d'un employeur payés à l'heure ou à la pièce. Ce dernier refuse de discuter le projet de contrat soumis par l'union. $\mathrm{La}$ Commission de relations ouvrières le somme de rencontrer les représentants de l'association ouvrière pour discuter un projet de contrat. Le cas est référé plus tard à un conciliateur. L'employeur déclare n'avoir aucune contreproposition à faire, vu qu'il ne reconnaît pas cette association comme agent négociateur et que la plupart de ses employés ont cessé leur adhésion à cette organisation. Le conciliateur explique la loi à l'employeur et lui conseille de voir son avocat. A la deuxième séance de conciliation, l'avocat accompagne l'employeur et tout marche bien.

Il arrive en d'autres cas que l'employeur persiste dans son attitude et alors un tribunal d'arbitrage sera formé. N'a-t-on pas vu un patron refuser de comparaître devant le tribunal d'arbitrage et préférer supnnrter une grève plutot que de négocier de bonne foi, une convention collective?

Les refus de négocier sont aujourd'hui des cas d'exception. Il est cependant plus fréquent que les deux 
parties en cause devant le conciliateur, ne veuillent pas se compromettre. Elles insisteront alors pour obtenir la formation d'un tribunal d'arbitrage. La décision de ce conseil, unanime ou majoritaire, servira alors aux agents négociateurs à se couvrir devant ceux qui les ont mandatés.

Ces difficultés portent sur la procédure de conciliation, elles sont donc d'un domaine différent de celui des problèmes soulevés durant la conciliation proprement dite.

Les parties ne s'entendent pas sur les salaires, les heures de travail, les congés annuels payés et les jours fériés et payés lorsqu'elles viennent en conciliation et personne ne s'en surprendra. Ce sont là, en effet, les clauses d'une convention collective qui font le plus souvent l'objet des séances de conciliation. Il est intéressant cependant d'étudier les tendances des associations ouvrières en matière de sécurité sociale.

Dans une grande proportion des projets de conventions collectives soumis par les associations ouvrières, on retrouve une clause prévoyant un plan d'assurance maladie-accidentvie. Ces plans sont loin d'être uniformes, voilà pourquoi nous nous attarderons à les étudier quelque peu.

Certaines associations ouvrières soumettront à l'attention du patron avec lequel elles sont en négociations, un plan d'assurance collective fourni par une des compagnies opérant dans la province de Québec. D'autres ont leur propre plan d'assurance approuvé par le Surintendant des assurances. Les contributions à ces projets d'assurance sont quelques fois payables uniquement par le patron alors qu'en d'autres cas employeurs et employés payent les primes dans une proportion qui, sans être uniforme dans tous les contrats, n'en est pas moins déterminée dans chacune des conventions.

Devant le conciliateur, deux problèmes principaux peuvent se poser: dans le premier cas, l'employeur refuse de contribuer à un plan d'as. surance. A force de discussion, il peut être possible de l'amener à accepter au moins le principe de sa contribution à un plan d'assurance et à fixer une certaine période au cours de laquelle les parties poursuivront l'étude de cette question, dans le but d'en venir à une entente.

Un problème plus ardu pour le conciliateur, se présente lorsque les deux parties ont chacune un plan d'assurance à se soumettre, ou lorsqu'un plan d'assurance existe déjà dans l'usine. Ce cas s'est présenté à plusieurs reprises et lorsque le conciliateur n'a pu réussir à amener les parties à s'entendre, le conseil d'arbitrage chargé d'étudier le même problème n'a pas réussi à satisfaire les parties en cause. Ce qui rend ce problème si ardu est qu'il faut faire des recherches sur l'état matrimonial, l'âge et le salaire des employés et, également tenir compte de cortains principes de l'actuariat en faisant une comparaison des bénéfices offerts et des primes exigées.

Il y a quelques mois, l'arbitrage intéressant un établissement et une association ouvrière prenait fin. L'union soumettait un plan d'assurance alors que la compagnie désirait maintenir celui qui était déjà en force. La conciliation ayant été infructueuse, un conseil d'arbitrage se mit à la tâche. Il y eut près de 70 séan. ces publiques et privées et la plupart portaient sur un plan d'assurance sans compter les heures durant lesquelles les arbitres étudièrent, chacun de son côté, les nombreux exhibits produits. Dans leur sentence, les arbitres préférèrent ne pas se prononcer sur la question.

Chez les associations patronales on ne retrouve pas, à proprement parler, de tendances généralisées. On note cependant une attitude nouvelle. La partie patronale n'a plus, la plupart du temps, d'objection à négocier, elle tient cependant à ce que l'association ouvrière justifie ses demandes. Le conciliateur n'en a pas moins 
de travail lorsque les parties sont devant lui parce qu'elles ont épuisé jusqu'alors un tas d'arguments pour soutenir leurs positions respectives. Il faut trouver des arguments nouveaux pour amener l'une ou l'autre à accepter un point de vue différent du sien.

Comme conclusion de ces quelques considérations sur des problèmes que le conciliateur rencontre, l'on ne peut qu'inviter les parties à coopérer. Cette attitude aura pour effet de rendre plus harmonieuses les relations patronales-ouvrières dans notre province. Actuellement un cas sur trois est soumis à l'arbitrage et cette proportion peut être réduite par une plus grande compréhension réciproque.

On peut être certain qu'en toutes circonstances, le conciliateur fera toujours son possible pour aider les parties à s'entendre. La conciliation de fait, n'a pas pour but de retarder les procédures d'arbitrage, mais bien de tenter d'apporter un règlement aux problèmes.

\section{II - LA CONCILIATION VUE DE L'INTERIEUR}

\section{par LÉOPOLD JASMIN}

Ce bref article n'a pas la prétention de définir la conciliation, ni d'en exposer les techniques. 'Tout au plus vise-t-il à souligner certains aspects d'un travail intéressant et utile qui se situe en plein centre de ce que les sociologues groupent sous le titre général de « relations humaines ».

On parle seulement de deux parties à un différend ouvrier-patronal. C'est prendre pour acquis qu'il y a identité d'intérêts du capital et de la gérance du côté patronal, èt également identité d'intérêts chez les travailleurs et les chefs syndicaux. En réalité, la situation est parfois plus complexe. La gérance peut attacher plus de poids à certaines de ses prérogatives qu'aux taux des salaires, tandis que les détenteurs du capital voient dans toute augmentation des salaires une mesure qui peut trancher dans le vif de leurs profits. D'autre part, les employés sont surtout intéressés au salaire, tandis que leurs chefs peuvent ajouter à cet objectif des préoccupations d'organisation ou d'expansion syndicale. $\mathrm{Ce}$ manque d'unité au sein même de l'une ou l'autre des parties en différend peut être un facteur de plus grande difficulté ou, au contraire aider au règlement final, puisque du salaire, par exemple, peut s'échanger contre de la sécurité syndicale, ou des prérogatives de la gérance contre du salaire.

Un cas où il y a nécessairement subdivision des parties, c'est celui des différends concernant les conventions sous l'empire de la Loi de la convention collective (décrets). Le groupe patronal comprend plusieurs employeurs dont les problèmes, la mentalité, les entreprises diffèrent. Il en résulte des regroupements par région, grandeur de l'entreprise, etc., avec des répercussions chez la partie ouvrière. Là encore, cette complexité peut rendre la besogne du conciliateur plus facile ou plus difficile, selon le cas. Le fond de toute l'affaire consiste généralement en problèmes de concurrence qu'il s'agit d'équilibrer.

Le plus mauvais service que les parties puissent se rendre à ellesmêmes et rendre au conciliateur, c'est de constituer un comité de négociation trop nombreux. Si chacun fait valoir son opinion, la discussion s'éternise. Dans le cas contraire, ceux qui ne se sont pas «vidés 》 au cours des séances se reprennent entre les séances en adoptant une attitude négative sur les compromis qui peuvent leur être soumis. Un groupe 